\title{
Hydrothermal synthesis of ZnSe nanoparticle
}

\author{
Xiaoguang Ren
}

Liaoning Jidian Polytechnic, Dandong, Liaoning 118009, China

48110551@qq.com

Keywords: hydrothermal, zinc selenide, nanomaterial, characterization

\begin{abstract}
The Zinc Selenide nanoparticle has been successfully synthesized with oleinic acid as surfactant by hydrothermal methord. Using X-ray diffractometer (XRD)、 scanning electron microscope (SEM)、transmission electron microscopy (TEM)、X-ray spectrometer (EDS)、 ultraviolet-visible spectrophotometer and other instruments on the product structure characterization and optical performance test. The results show that the prepared products were cubic blende ZnSe nanospheres, the average diameter of $20 \mathrm{~nm}$, good dispersion. And systematically discusses the surface active agent, reaction temperature, reaction time, $\mathrm{PH}$ and other process factors on the preparation of the morphology of ZnSe nano materials and the influence of grain size, etc.
\end{abstract}

\section{Introduction}

Recent years, nano semiconductor material has drawn more and more attention to the world due to its special and unique optical and electrical performance, as well as the great adoption and potential in nano material industry ${ }^{[1]}$. With the consistent development of semiconductor material, nano metallic selenides have become another interest to research on after oxides and sulfides ${ }^{[2-9]}$.

ZnSe is a very important II-VI semiconductor materials with a room temperature bulk band gap of $2.67 \mathrm{eV}^{[10]}$. ZnSe shows good optical transmission performance under light with wavelength range between $0.5 \sim 22 \mu \mathrm{m}$, basic coverage of the visible - infrared range, is an ideal material for the manufacture of photovoltaic devices. It can be used to produce blue light-emitting device ${ }^{\text {[11-12], }}$ transmission window of thermal infrared device and laser device, high density data storage device in communication and printing industry, high resolution display, signal indicator, as well as medical research use and many other applications over many kinds of industries.

\section{Experimental}

\section{ZnSe nanoparticle synthesis.}

In order to study the impact of oleic acid as a surfactant to the prepared nano zinc selenide morphology and particle size, in this experiment took I、 II two reactions on the same principle, but differ in the preparation process steps:

I (without oleinic acid): mixed $0.272 \mathrm{~g}$ zinc chloride with $5 \mathrm{ml}$ absolute ethylalcohol, heated under water at $60^{\circ} \mathrm{C}$ for 30 minutes and achieved transparent liquid A. Dissolved 0.1754 g sodium selenite with $5 \mathrm{ml}$ distilled water, then added $20 \mathrm{ml} 1 \mathrm{~mol} / \mathrm{L}$ sodium hydroxide solution and $5 \mathrm{ml}$ hydrazine hydrate, and obtained another transparent liquid B; Placed liquid A and B in autoclave, slowly heated it in oven till temperature reached between $120^{\circ} \mathrm{C} \sim 180^{\circ} \mathrm{C}$ and kept heating for certain time. After the reaction completed, naturally cooled it to the room temperature.

II (with oleinic acid): as experiment I has been stated, obtained liquid A. Dissolved 0.1754g sodium selenite with $5 \mathrm{ml}$ distilled water, mixed it with $5 \mathrm{ml}$ hydrazine hydrate and $8 \mathrm{ml} 1 \mathrm{~mol} / \mathrm{L}$ sodium hydroxide solution till transparent liquid B formed. Placed liquid B in autoclave and added 12 oleinic acid on top of the liquid $\mathrm{B}$, then placed liquid $\mathrm{A}$ in autoclave, sealed the autoclave and heated it in oven till temperature reached $120^{\circ} \mathrm{C} \sim 180^{\circ} \mathrm{C}$ then kept heating for certain time. After the reaction completed, naturally cooled it to the room temperature.

Washed the product by absolute ethylalcohol for several times, then dried it at $60{ }^{\circ} \mathrm{C}$ vacuum to get a pale yellow powdered zinc selenide sample. 


\section{Sample characterization.}

The phase structure and grain size of samples were identified by SHIMADZU -6000 series X-ray diffractometer. Morphologies of the ZnSe were observed by JEOL JSM-6360 LV SEM and FEI Tecnai G2 20 TEM. The absorption spectrum of ZnSe sample was investigated by JASCO V-550 UV-VIS spectrophotometer. Sample`s element category and content was analysed via Oxford INCA X-ray energy spectrometer.

\section{Results and Discussion}

\section{Phase composition analysis.}

Figure 1 is the experimental I (without oleic acid modified) under different water fluid temperature insulation $24 \mathrm{~h}$ zinc selenide was prepared by the X-ray diffraction spectrum of the sample. The diagrams show that all the samples' three main peaks respectively corresponding to (111), (220), (311), with the JCPDS card number in the database for 37-1463 cubic sphalerite ZnSe crystal diffraction pattern match exactly. The diffraction peak can be seen from the diagram, with the increase of hydrothermal temperature, the diffraction peak becomes narrow, which shows that the grain size of the powder is more complete, and the grain size increases. The B,C,D spectral lines that without impurity peaks suggest the object images of their ZnSe samples are pure. Meanwhile, the spectral line from A sample shows impurity peaks, judging from the weak peak, may suggest that the reaction could not go well under low temperature relatively.

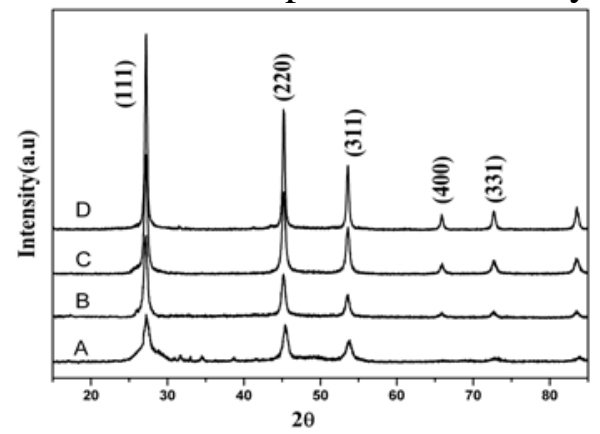

Fig $1 \mathrm{X}$-ray diffraction patterns of ZnSe samples heated at different temperature for 24 hours
A. $120^{\circ} \mathrm{C}$;
B. $140^{\circ} \mathrm{C}$;
C. $160^{\circ} \mathrm{C}$;
D. $180^{\circ} \mathrm{C}$

Figure 2 shows the EDS spectra of the Znse sample. The figure shows the sample after several times washing by absolute ethylalcohol, in the preparation of zinc selenide samples, only detected element zinc and the existence of Se, $\mathrm{Na}$ and $\mathrm{O}$ are not found in other elements, such as the preparation of sample with high purity. EDS characterization results correspond with the XRD results, further proved that the preparation of samples for high purity ZnSe.

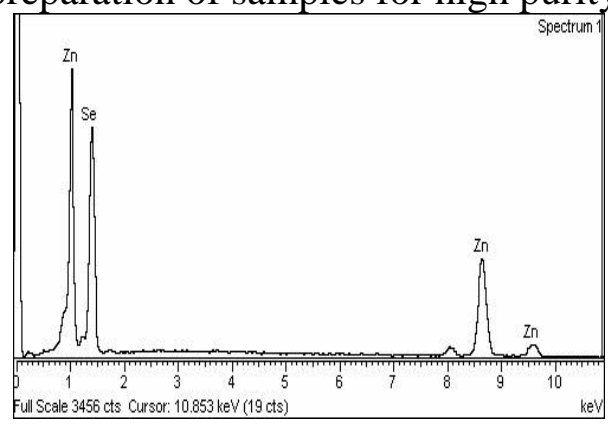

\section{Morphology analysis.}

Fig.2 X-ray energy spectroscopy of the ZnSe samples

Figure 3 shows experiment I (without olienic acid) the SEM image of ZnSe sample synthesized at $180^{\circ} \mathrm{C}$ with 10 hours heat preservation under different $\mathrm{PH}$ value enviroment. From the SEM image, it shows in Sample A, most of the crystalloid grains present with even size (60nm approximately), besides, also many small crystalloid grains form larger grains which are hundreds of nanoparticle in size, the agglomeration is relatively significant. In Sample B, the agglomeration grains formed by smaller crystalloid significantly reduced, comparing Sample A, the dispersity is 
improved, the crystalloid size increased as well (70nm approximately); In Sample C, the crystalloid forms globe shape, the dispersity looks well, the grain size is even due to no agglomeration happened.

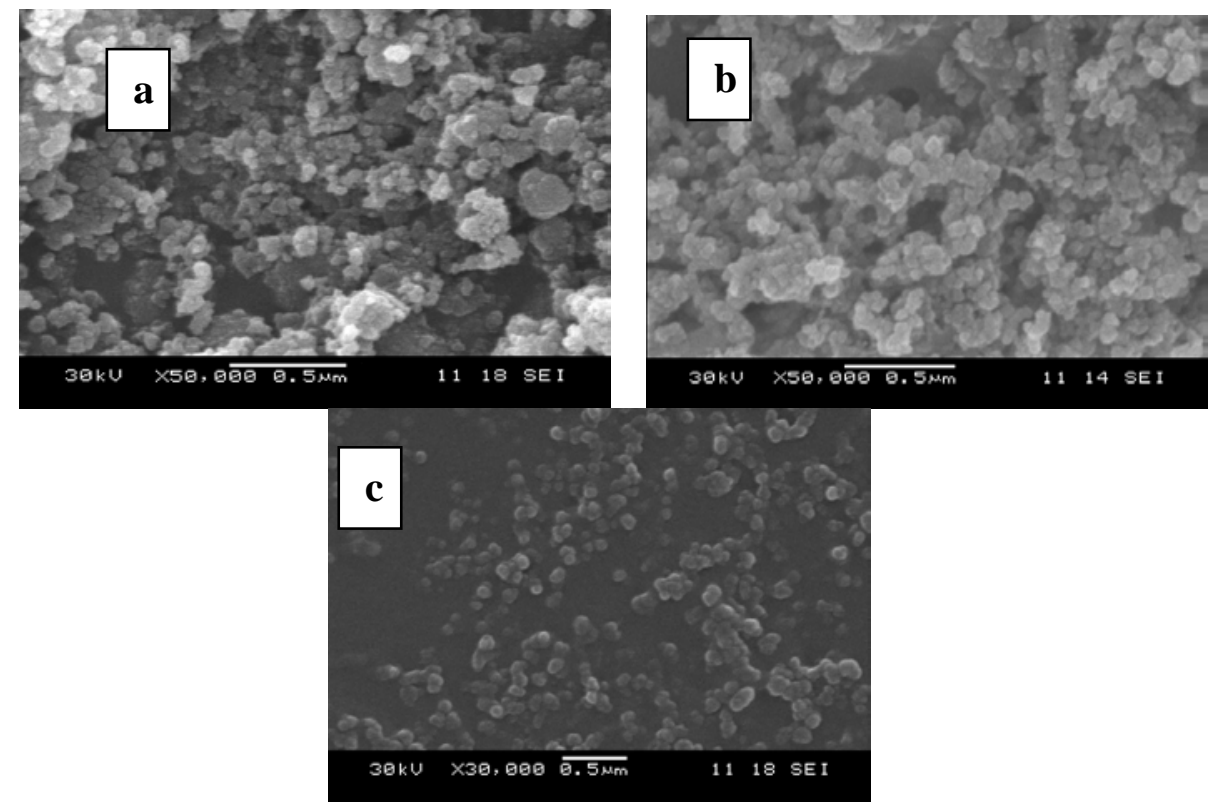

Fig.3 SEM images of ZnSe nanoparticles synthesis by experiment I .

(a) without $\mathrm{NaOH}$ solution $(\mathrm{PH}=10) \quad$ (b) with $12 \mathrm{ml} \mathrm{NaOH}$ solution( $\mathrm{PH}=12) \quad$ (c) with $20 \mathrm{ml} \mathrm{NaOH}$ solution( $\mathrm{PH}=14)$

Figure 4 shows experiment II (with olienic acid) TEM image of the sample ZnSe synthesized at $180^{\circ} \mathrm{C}$ with 10 hours heat preservation. From the image, the synthesized ZnSe nano grain forms globe shape with even size, the coalescence is relatively less significant, there is no large size grain formed due to coalescence, the grain size is evenly $20 \mathrm{~nm}$ which is reduced comparing experiment I. By the comparing Figure 3 and 4, it suggests that the olienic acid plays an important role in ZnSe grain size formation and dispersity.

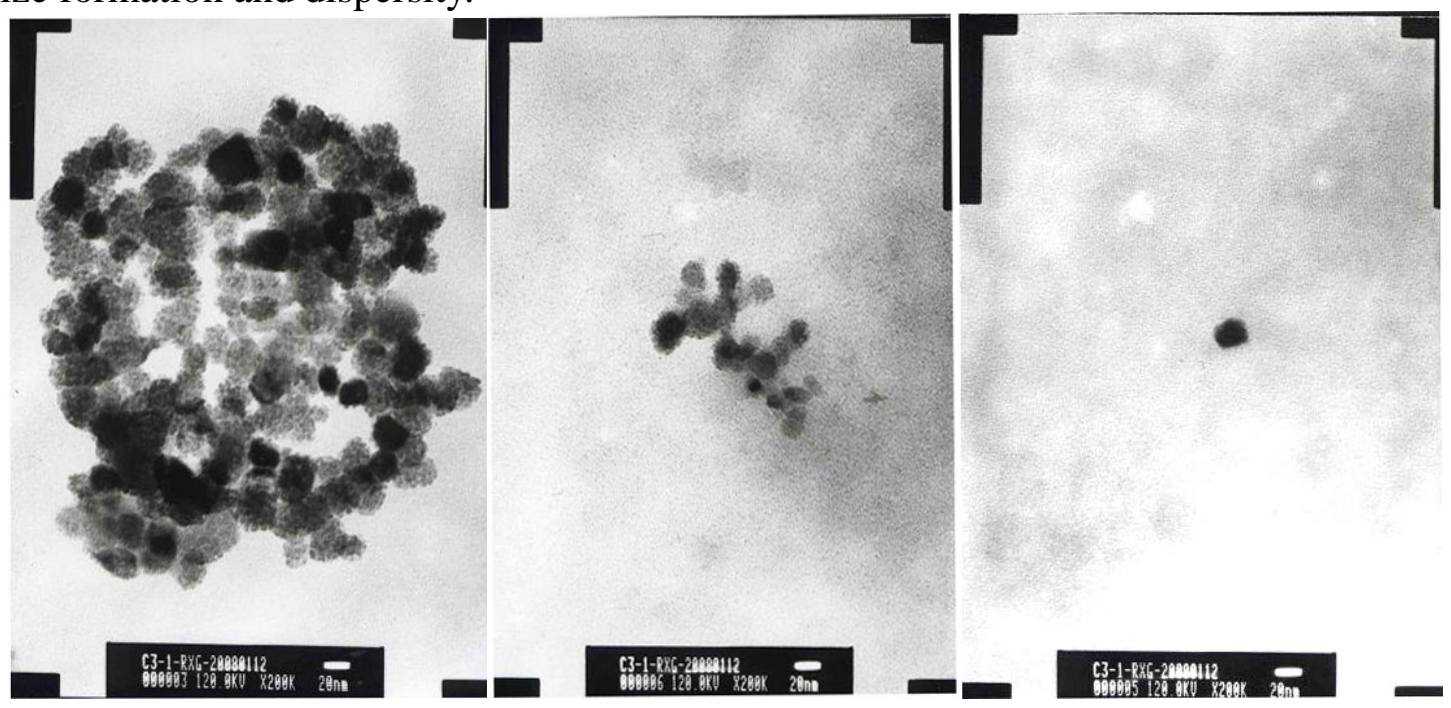

Fig.4 TEM images of ZnSe nanoparticles synthesis by experiment II

Factors that affects the object image of synthesized ZnSe.

(1)Surfactant effect

In Experiment II, the olienic acid was adopted as surfactant, affected by olienic acid, the ZnSe grain forms relatively smaller, decent, even shape in size. Inside the pressure pan, the olienic acid plays role as Figure 5 shows, the reaction solution $\mathrm{B}$ is composed with $\mathrm{Zn}^{2+}$ from $\mathrm{ZnCl}_{2}$ dissolved by ethylalcohol. As the ethylalcohol can dissolve each other with water or olienic acid (water and olienic acid can not dissolve each other), thus the $\mathrm{Zn}^{2+}$ dissovled in ethylalcohol can combine with the olienic acid $\left(\mathrm{C}_{17} \mathrm{H}_{33} \mathrm{COOH}\right)$ properly to form zinc oleate $\left(\mathrm{Zn}(\mathrm{RCOO})_{2}\right\rfloor^{[13]}$.Meanwhile, the $\mathrm{Zn}^{2+}$ performs strong solubility agaist polar solution (aqueous solution). This makes the complex 
$\left(\mathrm{Zn}(\mathrm{RCOO})_{2}\right)$ drift in Figure 5 to the border of "water-oil" which is formed by olienic acid and Solution A. Within the "water-oil" space, $\mathrm{Se}^{2-}$ is constantly realeased, and it reacts with $\mathrm{Zn}^{2+}$ from zinc oleate $\left(\mathrm{Zn}(\mathrm{RCOO})_{2}\right.$ ) together to form $\mathrm{ZnSe}$. The $\mathrm{RCOO}^{-}$forms RCOOH (olienic acid) instantly due to $\mathrm{Zn}^{2+}$ loss, then the $\mathrm{RCOOH}$ attache on the surface of the newly generated $\mathrm{ZnSe}$ grains. Due to the cover up by olienic acid, the ZnSe shows hydrophobicity when it was formed, this prevents the coalescence, also restricts the growing size of the grains. Eventually, the ZnSe settles at the bottom of the solution due to gravity and the fact that the olienic acid covered $\mathrm{ZnSe}$ can not be dissolved by water.

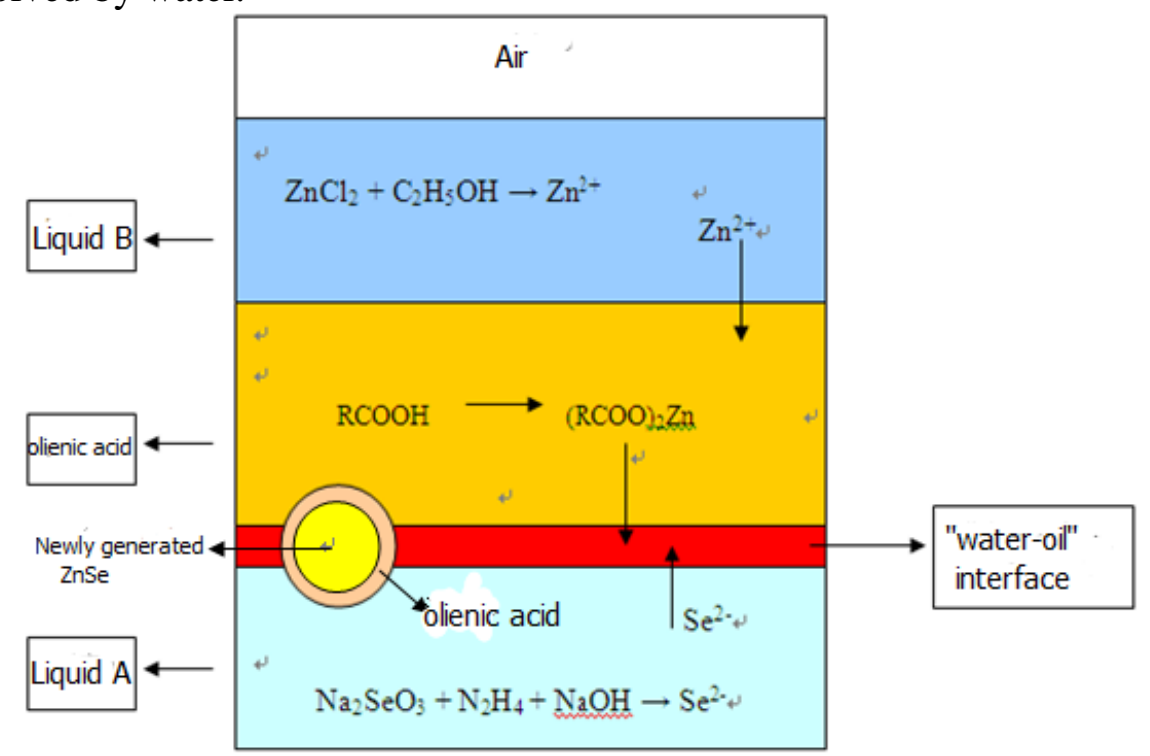

Fig.5 Oleic acid as surfactant zinc selenide nanoparticles reaction system

(2)Hydrothermal process duration effects

Under constant temperature at $180^{\circ} \mathrm{C}$, it shows the effects on the grains object image shape by the hydrothermal process duration of solution which is $\mathrm{NaOH}$ free, the SEM image in Figure 6. Reaction duration under 10 hours is shown from SEM image in Figure 3a.
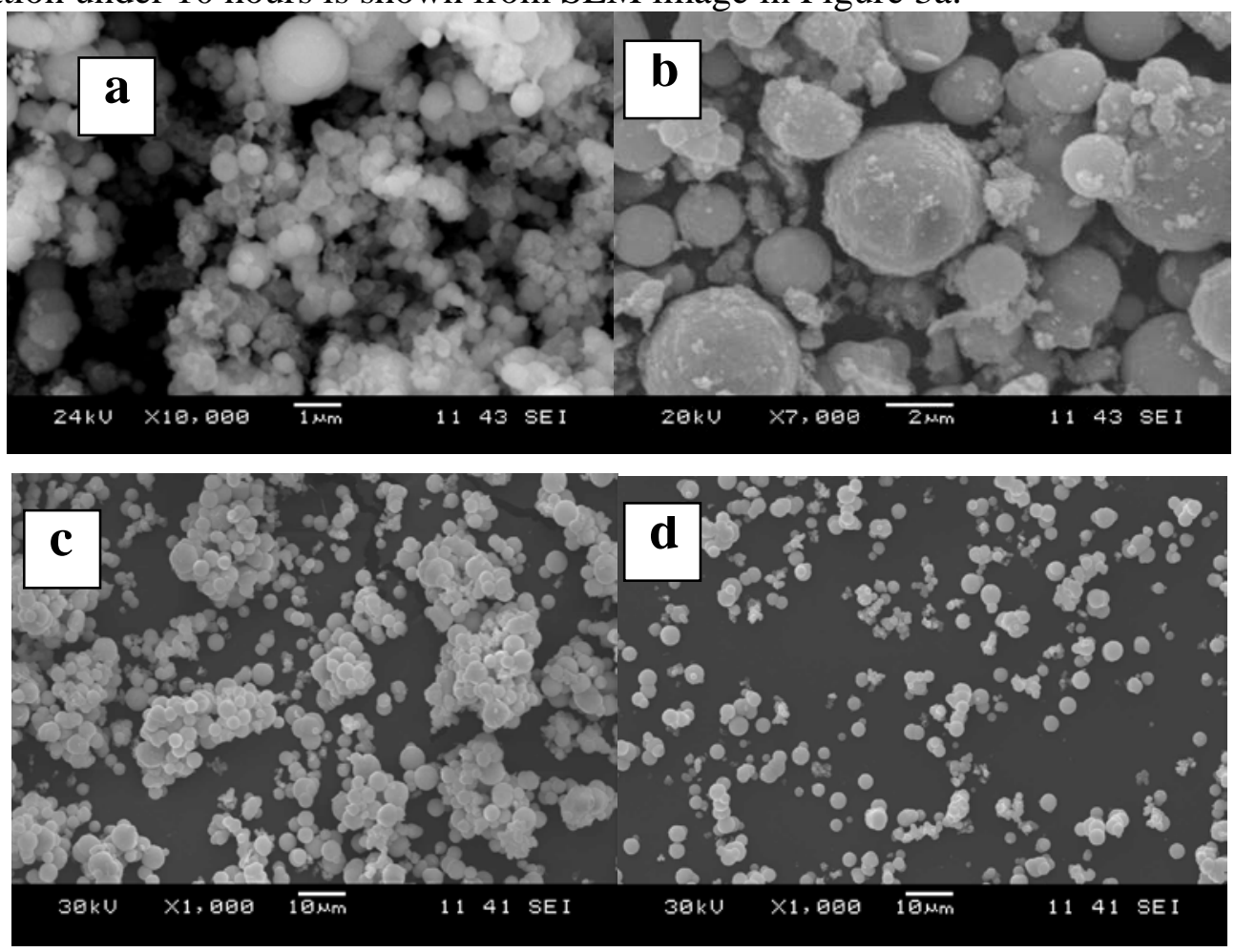

Fig.6 SEM images of ZnSe nanoparticles synthesized at $180^{\circ} \mathrm{C}$ for different reaction time (a) $14 \mathrm{~h} 、$ (b) $16 \mathrm{~h} 、$ (c) $18 \mathrm{~h} 、$ (d) $24 \mathrm{~h}$ 
By comparing the SEM images, as when the heat preservation duration inceased from 10 hours to 18 hours, the approximate size of the grains varies as $60 \mathrm{~nm} 、 300 \mathrm{~nm} \sim 1 \mu \mathrm{m} 、 2 \mu \mathrm{m} 、 3 \mu \mathrm{m}$. It suggests that when the hydrothermal duration is increased, the synthesized ZnSe grain size goes up, the coalescence is eased, the grain shape forms more regularly, the grain size falls more even. However, as the heat preservation duration is further increased, the grain size will not be changed any more, only the object image will become more subtle, and the dispersity will be improved even better, shows in Figure 6d.

(3)Reaction temperature effects

When the reaction duration is set to 24 hours, under same other circumstances, effects under different temperature separately at $120^{\circ} \mathrm{C} 、 140^{\circ} \mathrm{C} 、 160^{\circ} \mathrm{C} 、 180^{\circ} \mathrm{C}$ are shown in Figure 1 , the result shows when the temperature is below $120^{\circ} \mathrm{C}$, it will be hard for ZnSe nano grains to form, the product shows random peaks in the lines, the object image pres ents poor purity. Considering the result that the peaks go high and sharp when the temperature goes up, may come to a conclusion that when the temperature rises, the ZnSe grain size will become larger, the shape will be more subtle. Figure $6 \mathrm{~d}$ proves the point that with 24 hours heat preservation at $180^{\circ} \mathrm{C}$, the grains form shows globe shape, smooth surface, clean and compact, also accords with the peaks shape well.

(4) $\mathrm{PH}$ value effects

The experiment also recorded the effects by $\mathrm{PH}$ value change. The $\mathrm{NaOH}$ solution effect on the shape of the product is insignificant though, but it has certain impact on the dispersity and diameter of the grains. Shows in Figure 3, when certain amount of $\mathrm{NaOH}$ solution is added, $\mathrm{PH}$ value goes up, the coalescence of synthesized ZnSe nano grain eases, but the grain size goes up.

\section{Conclusions}

With the adoption of olienic acid as surfactant, operated through hydrothermal methord, reacted in "water-oil" based reaction zone, the ZnSe grains with even size, decent dispersity was successfully synthesized with blue shift presentation. The study also shows the complexes covered effects created importantly significant impact on the grain size and dispersity. Additionally, the reaction duration, reaction temperature, $\mathrm{PH}$ value also created significant impact on the object image and dispersity. The best experiment practise can be identified through optimization and comparison.

\section{References}

[1] Zeng H, Li J, Liu J.P.Exchange-coupled Nanocrystalites Mangnets by Nanoparticle Self-assemble [J].Nature,2002,420: 395-398

[2] Eitan Oksenberg,Ronit Popovitz-Biro,Katya Rechav,Ernesto Joselevich. ZnSe Nanowires: Guided Growth of Horizontal ZnSe Nanowires and their Integration into High-Performance Blue-UV Photodetectors[J]. Advanced Materials,2015,27: 3973

[3] Lingling Peng,Yuhua Wang, Qizheng Dong, Zhaofeng Wang. Passivated ZnSe nanocrystals prepared by hydrothermal methods and their optical properties[J]. Nano-Micro Letters,2010,2: 190-196

[4] Wang, Jun $\mathrm{Xu}$, Weining Su, Zhongyuan Ma and Kunji Chen. Sensitization enhancement of europium in ZnSe/ZnS core/shell quantum dots induced by efficient energy transfer[J]. LUMINESCENCE,2014,29: 1095-1101

[5] Kui Yu, CdSe Magic-Sized Nuclei, Magic-Sized Nanoclusters and Regular Nanocrystals: Monomer Effects on Nucleation and Growth[J]. Adv.Mater,2012,24: 1123-1132

[6] Jing Xu, Aijiang Lu, Chunrui Wang, et al. ZnSe-Based Longitudinal Twinning Nanowires[J].Advanced Engineering Materials,2014,16: 459-465 
[7] Li Y.D,Ding Y,Wang Z,Y.A Novel Chemical Route to ZnTe Semiconductor Nanorods[J].Adv.Mater,2000,12: 818-820

[8]Gaëlle Amiri, Ahmed Souissi, Nadia Hanèche, et al. Synthesis and characterization of core-shell $\mathrm{ZnO} / \mathrm{ZnSe}$ nanowires grown by MOCVD[J].PHYSICA STATUS SOLIDI (B),2013,250 : 2132-2136

[9] Valentina Zannier, Faustino Martelli, Vincenzo Grillo, et al. Strong blue emission from ZnSe nanowires grown at low temperature[J]. PHYSICA STATUS SOLIDI,2014,8: 182-186

[10] M. Nirmal, B.O. Dabbousi, M.G. Bawendi, J.J. Macklin, J.K. Trautman,T.D. Hattis, L.E. Brus. Fluorescence intermittency in single cadmium selenide nanocrystals [J].Nature,1996,383 (6603): 802-804.

[11]Arne G,Garsten K,Matthias K,et al. ZnSe-based laser diodesNew Approaches[J]. PHYSICA STATUS SOLIDI (C): conference,2005,2(3): 1098-1102

[12]E. Roventa1, R. Kröger, M. Klude, et al. Microstructural study of quantum well degradation in ZnSe-based laser diodes[J]. PHYSICA STATUS SOLIDI (C),2004,241（3） : 727-730

[13] Li Yadong, Liao Hongwei, Ding Yi, et al. Solvothermal elemental diret reaction to CdE ( $\mathrm{E}=\mathrm{S}$, Se, Te) semiconducto rnanocrystal[J].Inorg Chem,1999,38: 1382-1387 